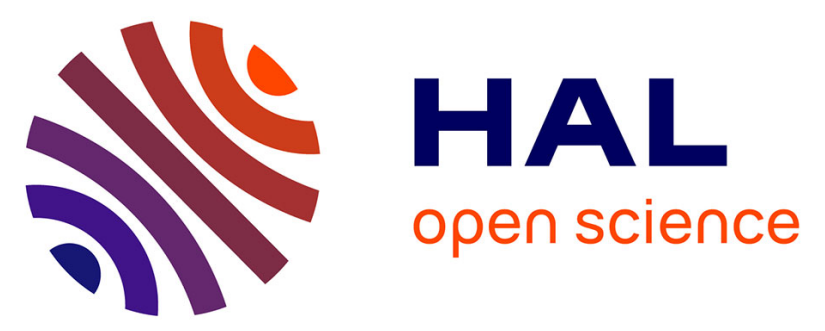

\title{
Single Event Transient Compact Model for FDSOI MOSFETs Taking Bipolar Amplification and Circuit Level Arbitrary Generation Into Account
}

Neil Rostand, Sébastien Martinie, Joris Lacord, Olivier Rozeau, Thierry Poiroux, Guillaume Hubert

\section{To cite this version:}

Neil Rostand, Sébastien Martinie, Joris Lacord, Olivier Rozeau, Thierry Poiroux, et al.. Single Event Transient Compact Model for FDSOI MOSFETs Taking Bipolar Amplification and Circuit Level Arbitrary Generation Into Account. 2019 International Conference on Simulation of Semiconductor Processes and Devices (SISPAD 2019), Sep 2019, Udine, Italy. 10.1109/SISPAD.2019.8870520 . hal02421069

\section{HAL Id: hal-02421069 \\ https://hal.science/hal-02421069}

Submitted on 8 Nov 2021

HAL is a multi-disciplinary open access archive for the deposit and dissemination of scientific research documents, whether they are published or not. The documents may come from teaching and research institutions in France or abroad, or from public or private research centers.
L'archive ouverte pluridisciplinaire HAL, est destinée au dépôt et à la diffusion de documents scientifiques de niveau recherche, publiés ou non, émanant des établissements d'enseignement et de recherche français ou étrangers, des laboratoires publics ou privés.

\section{(ㄷ)(1) $\$$}

Distributed under a Creative Commons Attribution - NonCommerciall 4.0 International 


\section{Single Event Transient Compact Model for FDSOI MOSFETs Taking Bipolar Amplification and Circuit Level Arbitrary Generation Into Account}

\author{
Neil Rostand \\ DPHY \\ ONERA \\ Toulouse, France \\ neil.rostand@onera.fr

Olivier Rozeau
DCOS
CEA-LETI
Grenoble, France
olivier.rozeau@cea.fr

\author{
Sébastien Martinie \\ DCOS \\ CEA-LETI \\ Grenoble, France \\ sebastien.martinie@cea.fr \\ Thierry Poiroux \\ DCOS \\ CEA-LETI \\ Grenoble, France \\ thierry.poiroux@cea.fr
}

\author{
Joris Lacord \\ DCOS \\ CEA-LETI \\ Grenoble, France \\ joris.lacord@cea.fr \\ Guillaume Hubert \\ DPHY \\ ONERA \\ Toulouse, France \\ guillaume.hubert@onera.fr
}

\begin{abstract}
Single Event Transients (SET) are ionizing particles induced current pulses which are able to generate soft errors in CMOS circuits. In Silicon-on-Insulator (SOI) technologies, bipolar amplification phenomena is more significant due to presence of the Burried Oxide (BOX), which is detrimental to soft errors sensitivity. State of the art FDSOI SET models account for bipolar amplification through a dynamic pre-factor. This approach is mainly empirical and not compact. In this work, we propose a SET compact model for FDSOI MOSFETs including a physical modeling of bipolar amplification. Results are validated through TCAD simulations. A circuit level approach is proposed considering arbitrary generation within functional SRAM cell. This approach allows more realistic Single Event Upset (SEU) prediction and we show how circuit level generation can influence SEU prediction.
\end{abstract}

Keywords- SET, Bipolar Amplification, SOI MOSFET, Compact Model, SPICE, TCAD.

\section{INTRODUCTION}

Soft errors in circuits are generally due to parasitic current induced by ionizing particles within MOSFETs, called Single Event Transients (SETs) [1]. In recent technologies based on SOI technology, bipolar amplification phenomena is more significant due the presence of the BOX and increase circuit sensitivity to single events [2-7]. It consists in parasitic source - drain current induced by storage of generated holes (in NMOS) or electrons (in PMOS) in the Silicon film. Another issue is the morphology of the particle induced charge deposit at the circuit level which matters for high level of integration as its spatial extension can overlap with many transistors volumes. Both circuit level charge deposit and bipolar amplification have to be taken into account in SET models in order to perform reliable soft errors risk assessment.

In the literature, the approach to model bipolar amplification in SOI technologies relies on consideration of equivalent access resistance to determine triggering of bipolar amplification. Classical SET current model (i.e without bipolar amplification; called $1^{\text {st }}$ discharge in [2]) is then multiplied by an empirical pre-factor [8]. This approach is not suitable for compact modeling (or SPICE modeling) point of view [9].

In this paper, we propose a compact model of SET taking both bipolar amplification and circuit level arbitrary charge deposit into account suitable for Fully Depleted SOI (FDSOI) structures (exposed for NMOSFETs). In Section. II, we evidence bipolar amplification through TCAD simulations of FDSOI MOSFET. In Section. III, we develop our bipolar amplification model and the Verilog-A implementation method. In Section. IV, we show the resulting SET compact model considering, $1^{\text {st }}$ discharge, bipolar amplification, and circuit level arbitrary charge deposit. The relevance of accounting for circuit level charge deposit is highlighted through SPICE simulations of Single Event Upsets (SEUs).

\section{EVIDENCE OF BIPOLAR AMPLIFICATION}

In this section, bipolar amplification is evidenced performing transient TCAD simulations [10] of a 2D FDSOI NMOSFET $\left(L_{c h}=0.1 \mu \mathrm{m}, T_{s i}=10 \mathrm{~nm}, V_{d s}=1\right.$, $V_{g s}=0 \mathrm{~V}$ ) with heavy ion strike considering simulation setup explained in [1]. After particle strikes the transistor, generated holes remain in the body (due to SOI structure) as illustrated in Fig. 1.a. This involves barrier lowering (see Fig.1.b) which allows electrons to flow from source to drain if $\mathrm{V}_{\mathrm{ds}}>0$, this extra current is the bipolar amplification. As illustrated in Fig. 1.c, after prompt $1^{\text {st }}$ discharges, we see these pulses exhibit relaxation tails which decay very slowly involving an higher collected charge at the drain $Q(t)=$ $\int I_{d} d t$ than deposited charge $Q_{d e p}$ which is the main feature of bipolar amplification. Fig 1.d illustrates the hole and electron quantity in the body (respectively denoted $P$ and $N$ ) for different $L E T$ versus time, and highlights that holes are stored inside the body after a short time interval corresponding to quick decay of $P$ (for high Linear Energy Transfer LET). We can actually show that this decay is due to quick recombination in source area. We also see electrons are injected in the body ( $\operatorname{ctill} N$ remains lower than $P$ ) in order to contribute to bipolar amplification current. 


\section{MODELIING OF BIPOLAR AMPLIFICATION}
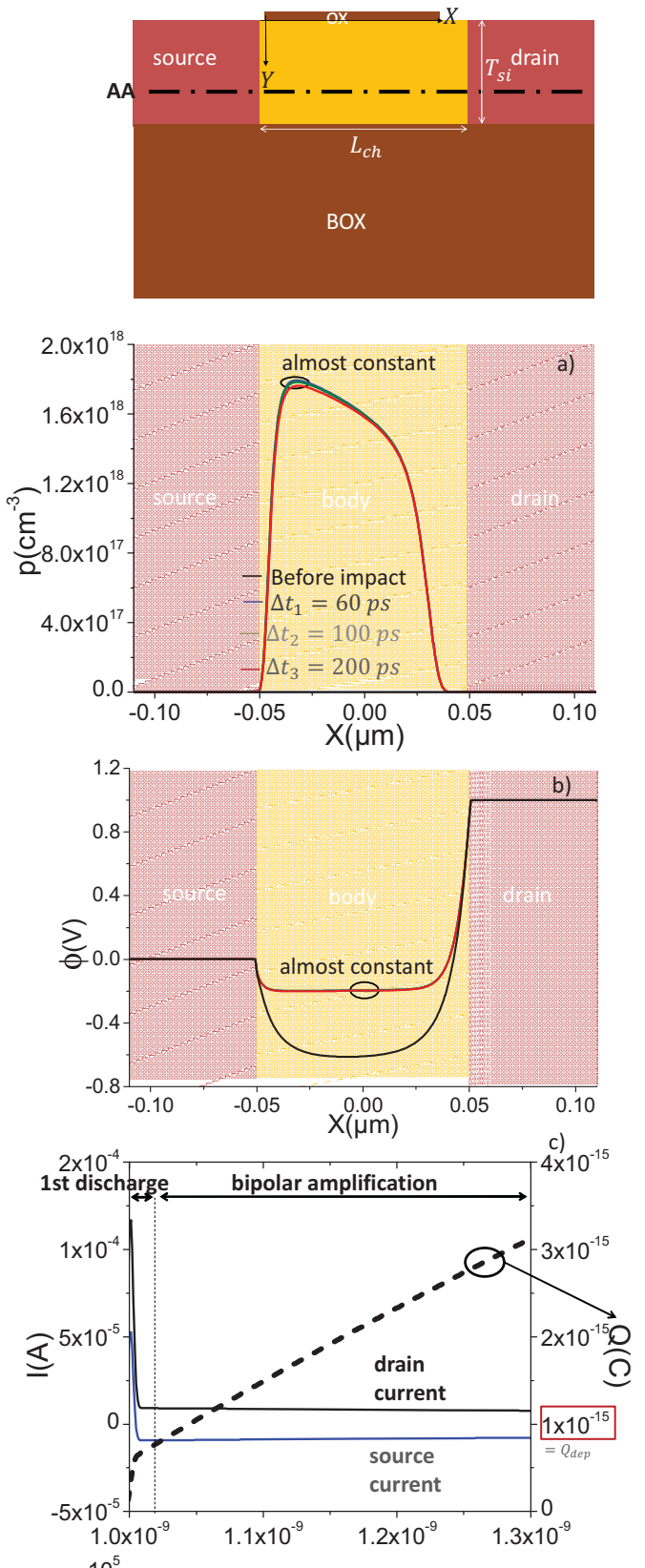

d)

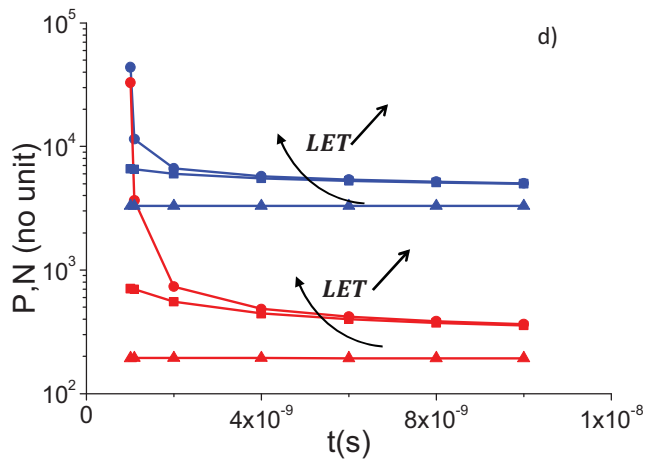

Fig. 1: TCAD evidence of bipolar amplification for $L E T=0.1 \mathrm{pC} / \mu \mathrm{m}$ $a, b)$ : plots of hole density $p$ and of the electrical potential $\phi$ in $A A$ cutline for different times after particle impact $\Delta t$. c): SET pulses at drain (black full line) and source (blue full line) electrodes and collected charge at the drain electrode (black dash line). d): quantity of holes (blue) and of electrons (red) relative to time after particle impact in the body. Circle, square, and triangle symbols correspond respectively to $L E T=\{1,0.1,0.05\} \mathrm{pC} / \mu \mathrm{m}$.

\section{A. Explicit bipolar amplification current expression}

In this part, we consider the particle generated $P_{\text {dep }}=L E T . T_{s i} / q$ electron/hole pairs in the body during impact at time $t_{i}$. We assume electron current density $\boldsymbol{J}_{\boldsymbol{n}}$ is conservative within the body, as evidenced by TCAD $\left(\boldsymbol{\nabla} \cdot \boldsymbol{J}_{\boldsymbol{n}} \approx 0\right)$. In a $1 \mathrm{D}$ problem along $X$, it means $\boldsymbol{J}_{\boldsymbol{n}}$ is uniform. Integrating $\boldsymbol{J}_{\boldsymbol{n}}$ in drift-diffusion formalism along $\boldsymbol{X}$, we obtained:

$j_{n}=-q \cdot \frac{\mu_{n}}{L_{c h}} \cdot \int_{0}^{L_{c h}} n \cdot \frac{\partial \phi_{n}}{\partial x} \cdot d x$

In (1), $\phi_{n}$ is the electron quasi-Fermi potential, $\mu_{n}$ the electron mobility in the body and $n$ the electron density. To capture $V_{d s}$ dependence, we consider simplified case of linear $\phi_{n}$ often called "long channel approximation". We can then derive (2), corresponding to source to drain bipolar amplification current:

$I_{b a}(t)=\frac{-q \cdot \mu_{n}}{L_{c h}^{2}} \cdot N(t) \cdot V_{d s}$

At this point we introduce the electroneutrality factor defined by $X(t)=N(t) / P(t)$. As the relation between time and $P$ is a bijection, we can redefine $X$ so that it is $P(t)$ dependant. Then:

$I_{b a}(P(t))=\frac{-q \cdot \mu_{n}}{L_{c h}^{2}} \cdot \mathrm{X}(P(t)) \cdot P(t) \cdot V_{d s}$

An empirical function is chosen for the electroneutrality factor $\mathrm{X}(P(t))$ (which is actually $L E T$ dependant). We now need to determine $P(t)$.

\section{B. Non-linear differential equation for $P(t)$}

Fig. 2 shows the hole quasi-Fermi potential $\phi_{p}$ in $X$ direction for different times after particle impact. We clearly see that $\phi_{p}$ is almost uniform in the body. Writing $d \phi_{p} / d x=0$ in the body, we obtain the $1^{\text {st }}$ order partial differential equation:

$\frac{\partial \Delta p}{\partial X}=-\frac{\partial \phi}{\partial X} \cdot \frac{1}{V_{t}} \cdot \Delta p$

In (4), $\Delta p$ is the excess hole density (generated by the particle), $\phi$ the electrical potential, and $V_{t}$ the thermic voltage. Considering $\Delta p$ property $\int_{0}^{L_{c h}} \Delta p \cdot d X \cdot W \cdot T_{s i}=$ $P(t)$ ( $W$ being the body width), the solution of this equation can be written as follow:

$\Delta p(X, t)=\frac{P(t)}{W \cdot T_{s i} \cdot I_{\phi}(t)} \cdot e^{-\frac{\phi(X, t)}{V_{t}}}$

Where $I_{\phi}(t)=\int_{0}^{L_{c h}} e^{-\frac{\phi(X, t)}{V_{t}}} \cdot d X$. The next step is to express the hole conservation law in the body, considering zero hole current at the source - body and body - drain junctions. However, surface recombination currents at these jonctions have to be taken into account because of high doping in source/drain areas. Note that no volume recombination occurs in the body for this time scale due to very low doping level $N_{a}=10^{15} \mathrm{~cm}^{-3}$. Denoting $V_{\text {rec }}$ for the recombination speed at the PN junctions, the resulting conservation law can be written as follow:

$\frac{d P}{d t}=-\frac{1}{\mathrm{~T}(P)} \cdot P$ 


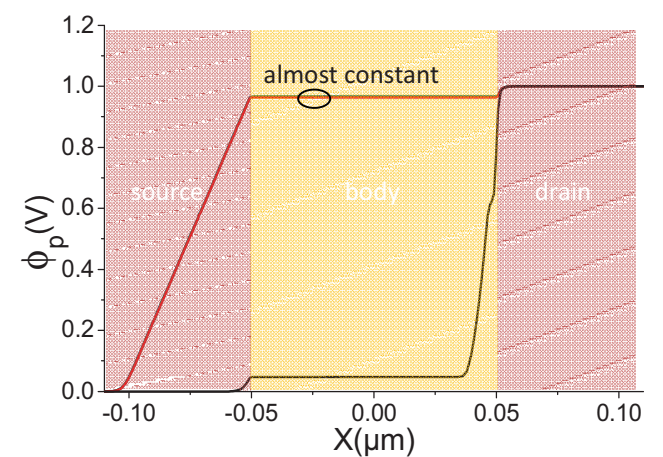

Fig. 2: plot of hole quasi Fermi potential $\phi_{p}$ in AA cutline for different times after particle impact $\Delta t$

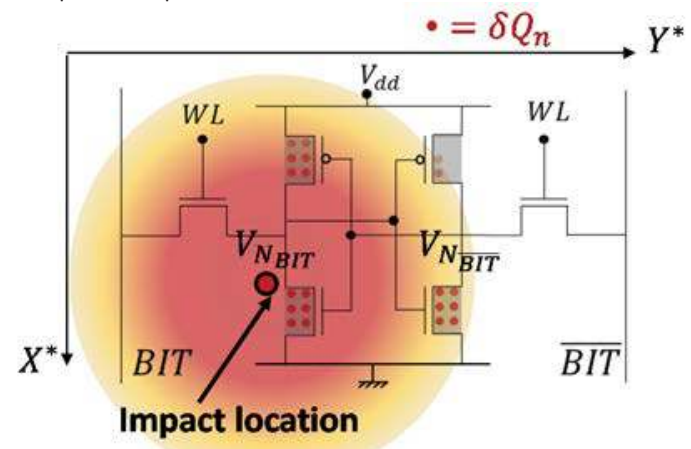

Fig. 3: 6T-SRAM cell submitted to particle strike. Illustration of discretization of circuit level charge deposit morphology induced by the particle in vertical incidence (orthogonal to the SRAM cell plane).

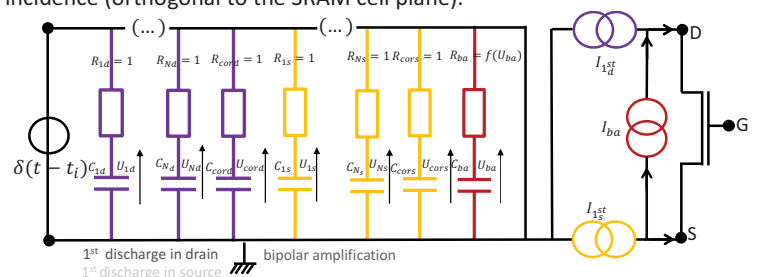

Fig. 4: equivalent electrical circuit of SET model in case of FDSOI. Modified $\mathrm{RC}$ circuit related to bipolar amplification completes initial $1^{\text {st }}$ discharge RC circuits, see [1,9].

$$
\mathrm{T}(P)=\frac{I_{\phi}(P)}{V_{\text {rec }}\left(e^{-\frac{V_{s}}{V_{t}}}+e^{-\frac{V_{d}}{V_{t}}}\right)}
$$

As the time constant (6.b) is not analytical, we prefer extract a simpler expression from TCAD, plotting the quantity $-P^{-1} \cdot d P / d t=\tau(P)^{-1}$. We obtain the following function:

$T_{e x}(P)=\tau_{r} \cdot\left(1+\left(\frac{P_{c}}{P}\right)^{\gamma}\right), \forall P>0, \gamma>0, P_{c}>0$

In (7), $\tau_{r}$ is the recombination time in source/drain while $\gamma$ and $P_{c}$ are parameters depending on geometry parameters (in particular $L_{c h}, T_{s i}$ and $W$ ) but this dependence has not been modeled. The set of implicit equations represented by (6.a) and (7) can be solved by the SPICE simulator, assigning $P / P_{d e p}$ to the voltage drop $U_{b a}$ of a capacitance being part of a modified RC circuit, where $\mathrm{R}$ value depends on $U_{b a}$, this circuit being submitted to $1 \mathrm{~V}$ voltage pulse at impact time of the particle. This equivalent circuit is implemented in Verilog-A.
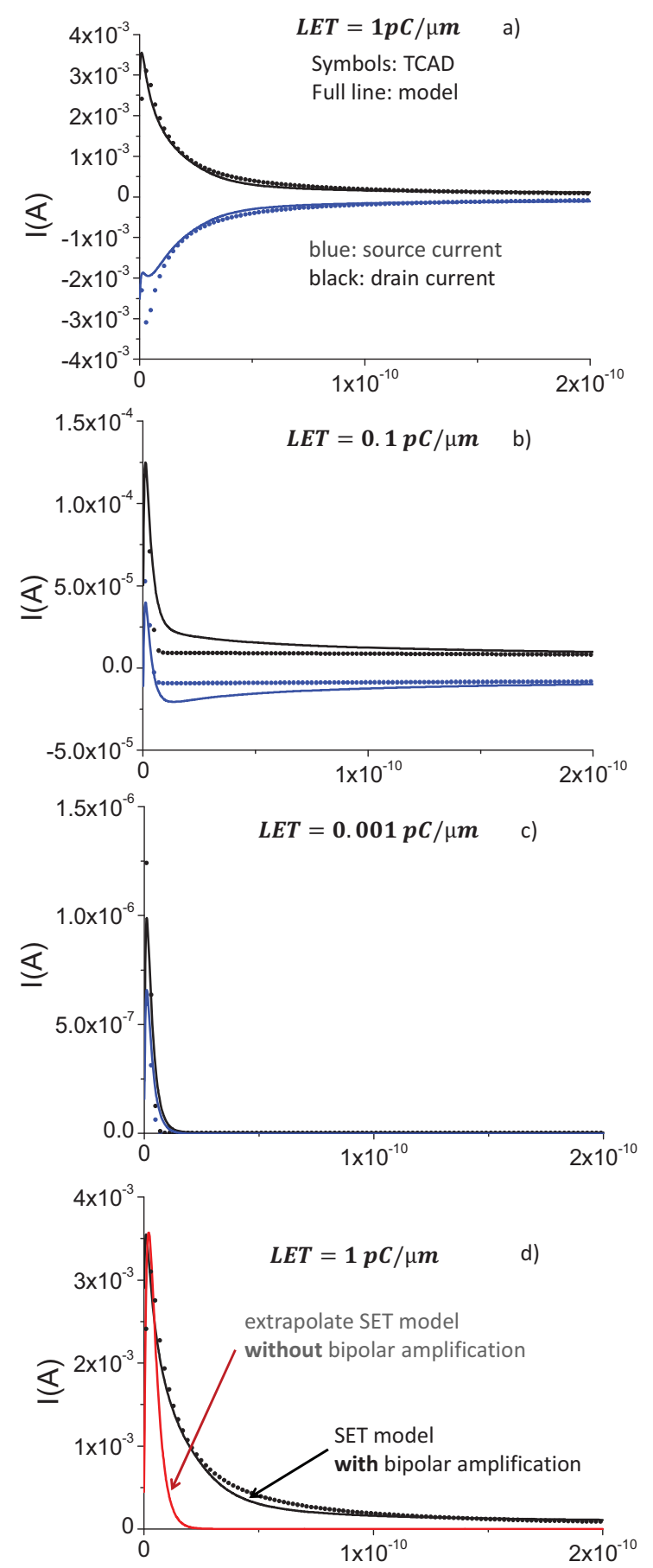

$\mathrm{t}(\mathrm{s})$

Fig. 5: a-c): comparison between TCAD and proposed SET model at source and drain electrodes for different LET values. Such a good agreement is obtained setting model parameters to consistent values: $D=$ 2.7.10 $10^{-4} \mathrm{~m}^{2} . \mathrm{s}^{-1}, v_{X}=4000 \mathrm{~m} \cdot \mathrm{s}^{-1}, D_{n}=2.25 .10^{-4} \mathrm{~m}^{2} \cdot \mathrm{s}^{-1}, P_{c}=$

$3.10^{3} \mathrm{C}, \gamma=3$. d): comparison between SET model with and without bipolar amplification and TCAD pulse at drain electrode after adjustment of model parameters for $L E T=1 \mathrm{pC} / \mu \mathrm{m}$ : we cannot describe the tail of the pulse.

\section{SET COMPACT MODEL FOR CIRCUIT LEVEL ARBITRARY CHARGE DEPOSIT: SEU PREDICTION IN SRAMS CELLS}

As bipolar amplification occurs after $1^{\text {st }}$ discharge, we can assume that $1^{\text {st }}$ discharge and bipolar amplification are independent. For $1^{\text {st }}$ discharge modeling, we improved 
former work [1,9] assuming the particle induced many punctual charge deposits $\delta Q_{i}$ at different locations of the circuit (made of many transistors) with coordinate $X_{i}^{*}$ along $X^{*}$, see Fig.3. We also consider uniform drift velocity $v_{X}$ in source - drain direction in the body of each transistor. For one given transistor of the circuit, the resulting $1^{\text {st }}$ discharge model at the drain for example is then:

$I_{1} s t(t)=\sum_{k=1}^{\infty} G_{k} \cdot e^{-\frac{t-t_{i}}{\tau_{k}}} \cdot H\left(t-t_{i}\right)$

$G_{k}=-q \cdot D_{n} \cdot T_{s i} \cdot W \cdot \frac{\partial A_{k}}{\partial X}\left(L_{c h}\right)$

$A_{k}=\frac{2 \cdot\left(\sum_{i \in \text { body }} \delta Q_{i} \cdot \sin \left(\frac{k \cdot \pi}{L_{c h}} \cdot X_{i}\right) \cdot e^{\left.\frac{v_{X}\left(X-X_{i}\right)}{2 \cdot D}\right)} \cdot \sin \left(\frac{k \cdot \pi}{L_{c h}} \cdot X\right)\right.}{W \cdot T_{s i} \cdot L_{c h} \cdot q}$

$\tau_{k}=\left(\frac{\pi^{2} \cdot k^{2} \cdot D}{L_{c h}^{2}}+\frac{v_{X}^{2}}{4 . D}\right)^{-1}$

In (8.a-d), $H$ is the Heaviside function, $D_{n}$ the electron diffusivity in the body, $X_{i}=X_{i}^{*}-X_{s}^{*}\left(X_{s}^{*}\right.$ being the source - body junction along $\left.X^{*}\right), X=X^{*}-X_{S}^{*}$, and $D$ is the ambipolar diffusivity.

Resulting SET model at the drain of one given transistor is then the sum of $I_{1}$ st and $I_{b a}$. Similar model can be obtained for the source SET current. The corresponding equivalent electrical circuit is shown in Fig. 4. Note that at circuit level, the number of deposited holes in the body are expressed as $P_{d e p}=\sum_{i \in b o d y} \delta Q_{i} / q$. SET Model has been validated through extraction of source and drain TCAD pulses for one generation point in the middle of the channel. We obtain good agreement between TCAD and model, after realistic calibration of $1^{\text {st }}$ discharge parameters $D, D_{n}, v_{X}$ and bipolar amplification parameters $\tau_{r}, P_{c}, \gamma$, as shown in Fig.5.a-c. Fig.5.d evidences the relevance of the bipolar amplification modeling work, comparing model with and without bipolar amplification.

We then apply the proposed SET model to SEU prediction performing transient SPICE simulations of standard 14nm FDSOI SRAM [11]. In Fig.6.a, we consider 2 different cases of charge deposit at $t=0$ : case 1 corresponds to deposit localized in non-sensitive PMOS for the considered initial bit state and case 2 to more realistic charge sharing with sensitive NMOS latch. We see that circuit level generation can influence SEU prediction as shown in Fig.6.b as a slight charge generation in sensitive NMOS latch can overcome large generation in non-sensitive PMOS (the latter actually reinforcing the initial state) and trigger the bit state flipping.

\section{CONCLUSION}

In this paper, we proposed a compact model of SET taking bipolar amplification and circuit level arbitrary charge deposit into account. Such a model is dedicated to FDSOI structures (FDSOI MOSFET, FinFET ...) and is suitable for performing realistic soft error risk assessment. TCAD simulations supported model development. More extensive work will be dedicated to improve predictability of the model focusing on electroneutrality function and description of the triggering of bipolar amplification around impact time.
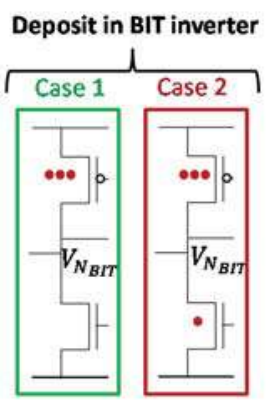

initial state:

$V_{N_{B I T}}=1 V$

$V_{N_{B I T}}=0 \mathrm{~V}$

$\stackrel{\bullet}{=} \delta Q_{n}=1.5 \mathrm{fC}$

b)

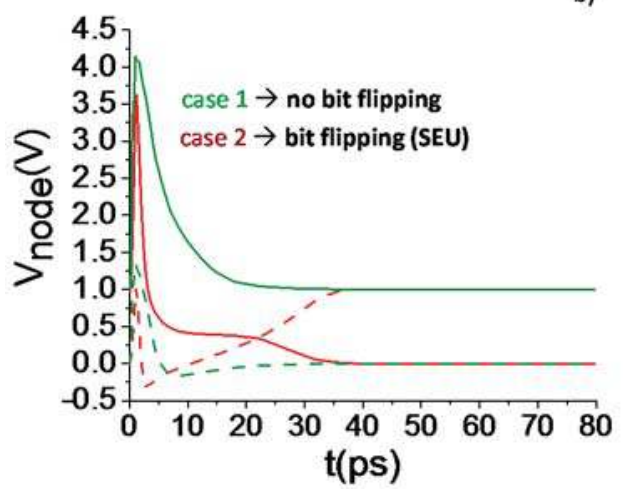

Fig. 6:a): illustration of 2 different cases of particle induced charge deposit at initial time within the BIT inverter b): dynamics of $V_{B I T}$ and $V_{\overline{B I T}}$ after particle strike for these cases: SEU is able to occur even if most of the charge is deposited in non-sensitive PMOS because of some charge deposited in sensitive NMOS Latch.

\section{REFERENCES}

[1] N. Rostand, S. Martinie, J. Lacord, O. Rozeau, J. Barbe and G. Hubert, "Single event transient in bulk MOSFETs: Original modelling for SPICE application," 2017 International Conference on Simulation of Semiconductor Processes and Devices (SISPAD), Kamakura, 2017, pp. 89-92.

[2] D. Kobayashi, M. Aimi, H. Saito and K. Hirose, "Time-Domain Component Analysis of Heavy-Ion-Induced Transient Currents in Fully-Depleted SOI MOSFETs," in IEEE Transactions on Nuclear Science, vol. 53, no. 6, pp. 3372 3378, Dec. 2006.

[3] M. Gaillardin et al., "Transient Radiation Response of Single- and MultipleGate FD SOI Transistors," in IEEE Transactions on Nuclear Science, vol. 54, no. 6, pp. 2355-2362, Dec. 2007.

[4] J. L. Autran and D. Munteanu - "Soft errors: from particle to circuits" CRC press Taylor \& Francis book 2015

[5] V. Ferlet-Cavrois et al., "Characterization of the parasitic bipolar amplification in SOI technologies submitted to transient irradiation," in IEEE Transactions on Nuclear Science, vol. 49, no. 3, pp. 1456-1461, June 2002.

[6] V. Ferlet-Cavrois et al., "Charge enhancement effect in NMOS bulk transistors induced by heavy ion Irradiation-comparison with SOI," in IEEE Transactions on Nuclear Science, vol. 51, no. 6, pp. 3255-3262, Dec. 2004.

[7] V. Ferlet-Cavrois et al., "Direct measurement of transient pulses induced by laser and heavy ion irradiation in deca-nanometer devices," in IEEE Transactions on Nuclear Science, vol. 52, no. 6, pp. 2104-2113, Dec. 2005.

[8] L. Artola, G. Hubert and R. D. Schrimpf, "Modeling of radiation-induced single event transients in SOI FinFETS," 2013 IEEE International Reliability Physics Symposium (IRPS), Anaheim, CA, 2013, pp. SE.1.1-SE.1.6.

[9] N. Rostand et al., "Compact Modelling of Single Event Transient in Bulk MOSFET for SPICE: Application to Elementary Circuit," 2018 International Conference on Simulation of Semiconductor Processes and Devices (SISPAD), Austin, TX, 2018, pp. 364-368.

[10] TCAD Sentaurus Device Manual, Synopsys, Inc.: J-2014.09

[11] R. Berthelon et al., "Investigation of SiGe channel introduction in FDSOI SRAM cell pFET and assessment of the Complementary-SRAM," 2018 Joint International EUROSOI Workshop and International Conference on Ultimate Integration on Silicon (EUROSOI-ULIS), Granada, 2018, pp. 1-4. 